\title{
The Management of Anticoagulants in Patients With Atrial Fibrillation and History of Falls or Risk of Falls: The Liverpool Af-falls Project. Protocol for a Systematic Review and Meta-analysis
}

\section{Thibaut Galvain ( $\nabla$ t.galvain@2019.ljmu.ac.uk)}

Liverpool John Moores University https://orcid.org/0000-0002-0412-249X

\section{Ruaraidh Hill}

Liverpool Reviews and Implementation Group, Department of Health Data Science, University of Liverpool and the Royal Liverpool and Broadgreen University Hospitals, Liverpool Health Partners, Liverpool

\section{Sarah Donegan}

Department of Health Data Science, University of Liverpool, Liverpool

\section{Paulo Lisboa}

Scool of Computer Science and Mathematics, Liverpool John Moores University, Liverpool

Gregory Y. H. Lip

Liverpool Center for Cardiovascular Science, Liverpool Heart and Chest Hospital, Liverpool

\section{Gabriela Czanner}

School of Computer Science and Mathematics, Liverpool John Moores University

\section{Protocol}

Keywords: Systematic review, meta-analysis, VKA, NOAC, falls, Atrial fibrillation, anticoagulant

Posted Date: July 1st, 2021

DOl: https://doi.org/10.21203/rs.3.rs-638316/v1

License: (a) This work is licensed under a Creative Commons Attribution 4.0 International License. Read Full License 


\section{Abstract}

\section{Background}

Atrial fibrillation affects an estimated 33 million individuals worldwide and is a major cause of stroke, heart failure, and death. Anticoagulants substantially reduces risk of stroke but are also associated with an increased risk of bleeding and especially intracranial hemorrhages which are the most feared complication. Because of this many patients are not offered anticoagulants, particularly patients at risk of falls or with history of falls. It is unclear what anticoagulant treatment these patients should be offered, and the Liverpool AF-Falls project aims to investigate this area. This protocol for a systematic review and meta-analysis aims to define what is the most appropriate anticoagulant treatment option for the for the management of atrial fibrillation patients at risk of falls or with a history of falls.

Methods

This systematic review and meta-analysis will include randomized and non-randomized studies evaluating safety and efficacy of different anticoagulant treatments (vitamin $\mathrm{K}$ antagonist, non-vitamin $\mathrm{K}$ antagonist oral anti-coagulant, anti-platelet agent and no treatment). Bibliographic databases (Cochrane Central Register of Controlled Trials, MEDLINE, Embase, Scopus, CINAHL, Web of Science and ClinicalTrials.gov) will be searched according to a pre-specified search strategy. Titles, abstracts, and full texts will be assessed by two independent reviewers and disagreement resolved with a third independent reviewer. The Cochrane Risk of Bias tool 2 will be used to assess risk of bias in randomized trials and the Newcastle-Ottawa-Scale tool will be used for non-randomized studies. Pairwise meta-analysis based on the fixed and random-effects models will be conducted. Publication bias will be evaluated with a funnel plot and the Egger's test. Heterogeneity will be assessed with the $I^{2}$ statistic. If conditions for indirect comparison are met and sufficient data are available, a network meta-analysis will be conducted using frequentist and Bayesian methodologies.

\section{Discussion}

This review will be the first to summarize direct and indirect evidence on safety and efficacy of anticoagulant treatments in atrial fibrillation patients at risk of falls or with history of falls. The findings will be important to patients, clinicians, and health policy-makers to inform best practice in the use of these treatments.

Systematic review registration

PROSPERO registry number: CRD42020201086

\section{Background}

Atrial fibrillation (AF) is the most common sustained cardiac arrhythmia (1) and is a major cause of stroke, heart failure, and death (2). Stroke is the second most common cause of death and it is a major 
cause of disability (3). AF patients have a yearly risk of stroke of $5 \%$, and this risk is increased in the presence of certain risk factors, including left ventricular dysfunction, hypertension, a history of stroke, and increasing age (4).

Treatment with oral anticoagulants substantially reduces risk of stroke but are also associated with an increased risk of bleeding and especially intracranial hemorrhages which are the most feared complication $(5,6)$. Because of that, many patients do not receive anticoagulants, and particularly patients at risk of falls or with history of falls (7). For instance, in a French cohort of older people resident in nursing homes with AF and at high risk of stroke, less than $50 \%(541 / 1085)$ received anticoagulant treatment. Recurrent falls (47\%), cognitive impairment (22.6\%), and advanced age (16.4\%) were the main reasons for not prescribing anticoagulants (8). However, Donzé et al. showed that patients on oral anticoagulants at high risk of falls $(\mathrm{n}=308)$ did not have a significantly increased risk of major bleeds than patients at low risk of falls $(n=207)$ (hazard ratio 1.09; 95\% confidence interval, 0.54-2.21) (9). Additionally, in elderly patients with AF the considerable stroke risk without oral anticoagulant (OAC) often exceeds the bleeding risk on OAC (10).

There are two classes of anticoagulant treatments : (i) vitamin $\mathrm{K}$ antagonist (VKA) and (ii) non-vitamin $\mathrm{K}$ antagonist oral anticoagulant (NOAC). Anticoagulant treatments are recommended in most patients at risk of strokes except in those with low risk of strokes. Alongside these two classes, antiplatelet agents are also prescribed despite not being recommended for stroke prevention in AF patients, regardless of stroke risks (11). In a recent meta-analysis in older AF patients, NOACs were associated with superior efficacy in preventing stroke/systemic embolism (HR 0.83, 95\% Cl: 0.74-0.94) and non-inferiority safety for major bleeding (HR 0.93, 95\% Cl: 0.86-1.01), intracranial bleeding (HR 0.58, 95\% Cl: 0.50-0.67) and gastrointestinal bleeding (HR 1.17, 95\% Cl: 0.99-1.38) compared to VKAs (12). Due to the perceived risks of bleeding, many patients are not offered anticoagulants, particularly patients at risk of falls or with history of falls. Thus, it is unclear what anticoagulant treatment these patients should be offered, and the Liverpool AF-Falls project aims to investigate this area.

This protocol for a systematic review and meta-analysis aims to define what is the most appropriate anticoagulant treatment option for the management of AF patients at risk of falls or with a history of falls.

\section{Research question and objectives}

This review will explore the following research question: What is the most appropriate anticoagulant treatment strategy for AF patients at risk of falls or with history of falls?

The objective of this systematic review and meta-analysis is to determine the safety and efficacy of different anticoagulant treatments for AF patients at risk of falls or with history of falls.

\section{Methods}


The protocol has been registered in the International Prospective Register of Systematic Reviews (PROSPERO) database and assigned an identifier CRD42020201086. The methodology used for this systematic review follows the recommendations of the Cochrane Handbook for Systematic Reviews of Interventions (13). The protocol is reported according to the Preferred Reporting Items of Systematic Reviews and Meta-Analysis for Protocols (PRISMA-P). The PRISMA-P checklist is available as an additional file (see Additional file 1). The review will be reported according to PRISMA 2020 (14).

\section{Eligibility criteria}

\section{Study designs}

Randomized controlled trials (RCT) (including post hoc and ancillary analysis), quasi-randomized studies and observational (prospective, retrospective, case control and cohort studies) studies will be included. Animal studies, editorials, letters, case reports, reviews, case series, eminence-based opinions and conference abstracts will be excluded. Systematic reviews of interventions will be excluded but included studies from relevant systematic reviews will be assessed for inclusion.

\section{Types of participants}

We will include studies of adults (age 18 or older) patients with any forms of nonvalvular AF (paroxysmal, persistent or permanent) with history of falls or that are at risk of falls. Patients were defined at risk of falls if they had one of these criteria (15):

- prior history of falls;

- lower extremity weakness;

- poor balance;

- cognitive impairment;

- orthostatic hypotension;

- use of psychotropic drugs;

- severe arthritis;

- dizziness.

Studies including patients receiving ablation, cardioversion, or left-atrial appendage closure will be excluded as they represent minor and special subsets of patients.

\section{Interventions and comparators}

Eligible studies will include any of the following interventions: 
- Vitamin K antagonists

- Direct oral anticoagulants or non-vitamin $\mathrm{K}$ antagonist oral anticoagulants

- Antiplatelet agents (aspirin and/or clopidogrel)

Comparators will be any of the above interventions or the absence of treatment. Studies where patients receive combinations of treatments will be excluded.

\section{Types of outcome measures}

The primary efficacy outcome will be the composite of ischemic stroke and/or systemic embolism (an acute vascular occlusion of an extremity or organ). The primary safety outcome will be major bleeding (defined based on International Society on Thrombosis \& Haemostasis for major bleeding in non-surgical patients (16)). Secondary outcomes will include:

- Intracranial hemorrhage (Including all intracerebral, subdural, epidural, subarachnoid hemorrhage and hemorrhagic stroke)

- Gastrointestinal bleeding

- Clinically relevant non-major bleeding (defined based on International Society on Thrombosis \& Haemostasis for major bleeding in non-surgical patients (17))

- Myocardial infarction

- Ischemic stroke

- Systemic embolism

- Hemorrhagic stroke

- Cardiovascular mortality

- All-cause mortality

\section{Search methods for identification of studies}

\section{Database searches}

The following bibliographic databases will be searched: Cochrane Central Register of Controlled Trials (CENTRAL); MEDLINE (via OVID); Embase (via OVID); Scopus, CINAHL and Web of Science. We will also search the following trials register: the US National Institutes of Health Register (www.clinicaltrials.gov). Finally, we will double-check the reference lists of all the relevant studies and review the articles to identify additional relevant studies.

\section{Search strategy}


The search strategy for bibliographic databases was developed from the research question and implemented by a health sciences librarian with expertise in searches for systematic reviews. A combination of terms of medical subject headings (MeSH) and keywords will be used in the search strategy for MEDLINE through Ovid (see Additional file 2). For Embase, similar terms and search limits will be used. MeSH terms will be replaced with Emtree indexing terms and/or keywords, as appropriate. The search strategies for MEDLINE and Embase will be adapted for use in Scopus, Web of Science and the other bibliographic databases. The search results will be entered into the EndNote X8 reference management software for screening, once duplicate records are removed.

\section{Data collection and analysis}

\section{Selection of studies}

Two stages of screening will be performed. Stage 1 screening will be conducted on the title and abstract of each citation to identify potentially eligible studies, applying the inclusion and exclusion criteria. Screening will be performed independently by two reviewers (TG and GC), with any questions resolved by consultation with a third reviewer (GL). All potentially eligible studies will be sought in full text for further screening and extraction. Stage 2 , the full text will be evaluated independently by two reviewers (TG and GC) against the inclusion and exclusion criteria, with questions or discrepancies resolved by consultation with a third reviewer (GL). The reason for exclusion will be noted for all articles rejected at stage 2 . Study authors will be contacted in cases further information is needed to make a screening decision. A PRISMA flow diagram will be developed to record the study selection process (14).

\section{Data extraction and management}

A custom data extraction template will be designed for this review in Microsoft Excel and used for all eligible studies. Data will be extracted from each eligible study by one reviewer (TG) and cross-checked with the source article by a second reviewer (GC). Discrepancies and differences in interpretation will be resolved through discussion, and if necessary, by consultation with a third reviewer ( $\mathrm{PL}$ or GL). Where insufficient data are presented, we will request additional information from the study authors by email. The following will be collected from each study: study characteristics (publication year, authors, title, study objectives and study outcomes), study population (such as age, gender, and diagnostic criteria), study design, intervention and control details, and outcomes including point estimates per group, treatment effect, confidence intervals and any p-value with associated statistical test. For RCTs, outcomes data will be sought on an intent-to-treat basis preferentially (results for all randomized patients, regardless of what treatment they received); however, where this information is not available, data will be extracted as reported by authors. As-treated data may be analyzed if available. For observational studies, adjusted results will be preferred over non adjusted, when available. 


\section{Assessment of risk of bias in included studies}

In this systematic review, risk of bias in observational studies will be appraised with the NewcastleOttawa Scale (NOS) (18). Using this tool, studies are scored between 0 and 9 according to the quality of participant selection, comparability of groups, and outcome adjudication. Risk of bias assessment of randomized controlled trials (RCTs) will be conducted with the Cochrane's Risk of Bias 2 (RoB 2), where trials are scored as high, low, or unclear risk of bias within 5 domains: bias from the randomization process, bias due to deviations from intended interventions, bias due to missing outcome data, bias in measurement of the outcome, and bias in selection of the reported result (13). Risk of bias will be independently evaluated by two reviewers (TG and GC) and will resolve any disagreements by discussion. Unresolved disagreements will be resolved through discussion with a third senior reviewer (GL).

\section{Measures of treatment effect}

\section{Time to event data}

Study outcomes are expected to be reported as time to event data. We will record the number of participants per group and the hazard ratio between groups, with $95 \% \mathrm{Cl}$ and $\mathrm{p}$ value, as reported. If necessary, methods described by Tierney and al. could be used to retrieve hazard ratio and its uncertainty $(19,20)$.

\section{Dealing with missing data}

In order to address loss to follow-up, a complete case analysis (based on number of patients analyzed in included studies) will be conducted.

\section{Assessment of heterogeneity}

Heterogeneity across studies will be assessed both by visual inspection of the forest plots and a formal statistical test, using Cochran Q test and $\mathrm{I}^{2}$ statistic (21). An $\mathrm{I}^{2}$ up to $50 \%$ could suggest moderate-tosubstantial heterogeneity and more than $75 \%$ could suggest substantial heterogeneity. If high levels of heterogeneity are identified, i.e., $\left.\right|^{2}$ more than $50 \%$, likely sources of heterogeneity will be further assessed using subgroup analysis (see "Subgroup analysis" section) and meta-regression, as data permit.

\section{Assessment of risk of bias due to missing results}

If more than 10 studies are included in the meta-analysis, selective outcome reporting and publication biases will be explored using a funnel plot to quantify the potential presence of publication bias. The asymmetry will be evaluated with Egger's regression test (22). 


\section{Data synthesis}

Summary statistics (study counts and patient numbers by key study characteristics) will be prepared from the set of studies meeting eligibility criteria, in order to assess the available evidence. Data synthesis will be performed according to the study heterogeneity. If heterogeneity is low or minor $\left(1^{2} \leq\right.$ $25 \%)$, a fixed effect model will be used to pool the data; if heterogeneity is moderate-to-substantial $(25 \%<$ $\left.I^{2} \leq 75 \%\right)$, a random-effects model will be use instead, and if heterogeneity is substantial $\left(I^{2}>75 \%\right)$, a narrative synthesis will be conducted with tabular summaries of the extracted data and a forest plot without meta-analysis results (21). For the fixed effect model, the generic inverse variance method will be used. For the random-effects model, data will be pooled across studies using the DerSimonian and Laird model (23). Results of meta-analysis will be presented as pooled HRs with $95 \%$ Cls. Prediction interval will be generated to interpret random-effects meta-analysis, which provides a predicted range for the true treatment effect in an individual study (24). Individual study results and pooled estimates of treatment effect will be presented in tables and graphically using forest plots.

Following the pairwise meta-analysis, we will evaluate if it is appropriate to also conduct a network metaanalysis (NMA): (i) we will generate a network diagram to summarize the direct pairwise comparisons available, all potential comparisons being presented in Fig. 1; (ii) the homogeneity assumption of the evidence will be assessed by comparing potentially treatment effect-modifying, patient or trial characteristics, across trials that allocate the same two treatments and by generating quantitative measure of heterogeneity (25); (iii) the consistency assumption will be evaluated by comparing patient or trial characteristic across all trials in the NMA and by applying node-splitting to the NMA model (25); Should we proceed with a network meta-analysis, we will conduct a Bayesian analysis for time to event outcomes. Estimated treatment effects will be reported as hazard ratios with $95 \%$ confidence intervals. Treatment rankings will be estimated with associated confidence intervals.

Statistical analyses will be performed in R using RStudio version 4.0 .0 (meta, gemtc, and metafor packages (26)).

\section{Quality of evidence}

Two reviewers ( $T G$ and $G C$ ) will assess the quality of evidence with the Grading of Recommendations Assessment, Development and Evaluation (GRADE) system which considers study design, risk of bias, inconsistency of results, indirectness, imprecision and other factors $(27,28)$. Disagreements will be resolved by a third review author (PL or GL). Each study will be scored from very low, low, moderate to high quality of evidence using GRADEproGDT. Summary of the evidence will be presented in a 'Summary of findings' table. This will provide key information about the best estimate of the magnitude of effect, in relative terms and as absolute differences $(27,29)$. The following outcomes will be included in the summary of findings table:

- Ischemic stroke and/or systemic embolism 
- Ischemic stroke

- Hemorrhagic stroke

- Major bleeding

- Intracranial hemorrhage

- All-cause mortality

\section{Risk of bias between studies}

If possible, subgroup analysis will be carried out to identify heterogeneity sources as follows:

- Studies including patients at risk of falls (without history of falls) compared to studies including patients with history of falls

- Randomized clinical trials compared to observational studies

- Stratifying studies according to stroke risk evaluated with $\mathrm{CHA}_{2} \mathrm{DS}_{2}-\mathrm{VASc}$ score $\left(\mathrm{CHADS}_{2}\right.$ in older studies)

- Stratifying studies according to bleeding risk assessed with the HAS-BLED score as patients may have different safety outcomes.

Sensitivity analysis will be conducted to investigate the robustness of our finding. The analysis will be repeated including only studies identified as low risk of bias. In case of inconsistency, the study characteristics will be further reviewed.

\section{Discussion}

This systematic review and meta-analysis will synthesize the available evidence of the safety and efficacy of anticoagulant treatment used in patients with atrial fibrillation and at risk of falls or with history of falls. Additionally, if the network meta-analysis is feasible, this will add even more value and benefits to evidence informed practice and patient outcomes. Despite AF guidelines, stroke prevention through anticoagulation treatment for patients with atrial fibrillation and at risk of falls or with history of falls is still subject to wide variation among clinicians. These patients might not even receive anticoagulant treatment, secondary to a perceived higher risk of bleeding complications. These risks have been assessed, but conclusive data regarding the risk-benefit trade-off are elusive. Additionally, it remains unclear what anticoagulant treatment (if any) these patients would benefit the most of. To our knowledge, this is the first systematic review on this topic and this work would provide clinicians and policy makers with information on which to make evidence-based recommendations. The study results will be submitted for publication in a peer-reviewed journal and disseminated to the public in an accessible format.

\section{Abbreviations}


AF: Atrial fibrillation; OAC: Oral Anticoagulant; VKA: Vitamin K Antagonist; NOAC: non-vitamin K antagonist oral anticoagulant; HR: Hazard Ratio; Cl: Confidence Interval; PRISMA-P: Preferred Reporting Items of Systematic Reviews and Meta-Analysis for Protocols; RCT: Randomized Controlled Trial; MeSH: Medical Subject Headings; NOS: Newcastle-Ottawa Scale; NMA: Network Meta-Analysis

\section{Declarations}

\section{Ethics approval and consent to participate}

Not applicable.

\section{Consent for publication}

Not applicable.

\section{Availability of data and materials}

Data sharing is not applicable to this article as no datasets were generated or analyzed during the current protocol. Results will be reported according to PRISMA 2020. The dataset that will be generated and analyzed during the current study will be available from the corresponding author on reasonable request.

\section{Competing interests}

TG is an employee of Johnson and Johnson Medical. RH declares a financial - non-personal, non-specific interest, having delivered educational workshops on health economics, medicines management and HTA for cancer specialists supported by unrestricted sponsorship by the pharmaceutical industry and an industry association (March 2019). No fees received personally. Not specific to the topic of the review.

\section{Funding}

TG's PhD education program is funded by Johnson and Johnson Medical. The funder was not involved in the research, nor in any decisions taken by the authors.

\section{Authors' contributions}

TG is the guarantor. GL, PC, GZ, SD and TG conceived the study. TG, RH and GL contributed to the clinical background and methodology. TG, GC, SD and PL contributed to the analytical plan and the bias assessment approach. TG developed the criteria and drafted the protocol. All authors read, revised, and approved the final protocol. 


\section{Acknowledgements}

We would like to acknowledge Donna Burgess and Carolyn Benny, both librarians, for their useful reviews and expert advice.

\section{References}

1. Markides V, Schilling RJ. Atrial fibrillation: classification, pathophysiology, mechanisms and drug treatment. Heart. 2003 Aug;89(8):939-43.

2. Chugh SS, Havmoeller R, Narayanan K, Singh D, Rienstra M, Benjamin EJ, et al. Worldwide epidemiology of atrial fibrillation: a Global Burden of Disease 2010 Study. Circulation. 2014 Feb 25;129(8):837-47.

3. Marini C, De Santis F, Sacco S, Russo T, Olivieri L, Totaro R, et al. Contribution of atrial fibrillation to incidence and outcome of ischemic stroke: results from a population-based study. Stroke. 2005 Jun;36(6):1115-9.

4. Risk Factors for Stroke and Efficacy of Antithrombotic Therapy in Atrial Fibrillation: Analysis of Pooled Data From Five Randomized Controlled Trials. Archives of Internal Medicine. 1994 Jul 11;154(13):1449.

5. Staerk L, Sherer JA, Ko D, Benjamin EJ, Helm RH. Atrial Fibrillation: Epidemiology, Pathophysiology, and Clinical Outcomes. Circ Res. 2017 Apr 28;120(9):1501-17.

6. Katsanos AH, Schellinger PD, Köhrmann M, Filippatou A, Gurol ME, Caso V, et al. Fatal oral anticoagulant-related intracranial hemorrhage: a systematic review and meta-analysis. Eur $\mathrm{J}$ Neurol. 2018;25(10):1299-302.

7. Kakkar AK, Mueller I, Bassand J-P, Fitzmaurice DA, Goldhaber SZ, Goto S, et al. Risk profiles and antithrombotic treatment of patients newly diagnosed with atrial fibrillation at risk of stroke: perspectives from the international, observational, prospective GARFIELD registry. PLoS ONE. 2013;8(5):e63479.

8. Bahri O, Roca F, Lechani T, Druesne L, Jouanny P, Serot J-M, et al. Underuse of oral anticoagulation for individuals with atrial fibrillation in a nursing home setting in France: comparisons of resident characteristics and physician attitude. J Am Geriatr Soc. 2015;63(1):71-6.

9. Donzé J, Clair C, Hug B, Rodondi N, Waeber G, Cornuz J, et al. Risk of falls and major bleeds in patients on oral anticoagulation therapy. Am J Med. 2012;125(8):773-8.

10. Man-Son-Hing M, Nichol G, Lau A, Laupacis A. Choosing antithrombotic therapy for elderly patients with atrial fibrillation who are at risk for falls. Arch Intern Med. 1999;159(7):677-85. 
11. Kirchhof P, Benussi S, Kotecha D, Ahlsson A, Atar D, Casadei B, et al. 2016 ESC Guidelines for the management of atrial fibrillation developed in collaboration with EACTS. Eur Heart J. 2016 07;37(38):2893-962.

12. Grymonprez M, Steurbaut S, De Backer TL, Petrovic M, Lahousse L. Effectiveness and Safety of Oral Anticoagulants in Older Patients With Atrial Fibrillation: A Systematic Review and Meta-Analysis. Frontiers in Pharmacology. 2020;11:583311.

13. Higgins J, Thomas J, Chandler J, Cumpston M, Li T, Page M, et al. Cochrane Handbook for Systematic Reviews of Interventions version 6.2 (updated February 2021) [Internet]. Cochrane, 2021; Available from: www.training.cochrane.org/handbook

14. Page MJ, McKenzie JE, Bossuyt PM, Boutron I, Hoffmann TC, Mulrow CD, et al. The PRISMA 2020 statement: an updated guideline for reporting systematic reviews. BMJ. 2021 Mar 29;n71.

15. Steffel J, Giugliano RP, Braunwald E, Murphy SA, Mercuri M, Choi Y, et al. Edoxaban Versus Warfarin in Atrial Fibrillation Patients at Risk of Falling: ENGAGE AF-TIMI 48 Analysis. Journal of the American College of Cardiology. 2016 Sep 13;68(11):1169-78.

16. Schulman S, Kearon C, the SUBCOMMITTEE ON CONTROL OF ANTICOAGULATION OF THE SCIENTIFIC AND STANDARDIZATION COMMITTEE OF THE INTERNATIONAL SOCIETY ON THROMBOSIS AND HAEMOSTASIS. Definition of major bleeding in clinical investigations of antihemostatic medicinal products in non-surgical patients: Definitions of major bleeding in clinical studies. Journal of Thrombosis and Haemostasis. 2005 Apr 4;3(4):692-4.

17. Kaatz S, Ahmad D, Spyropoulos AC, Schulman S, the Subcommittee on Control of Anticoagulation. Definition of clinically relevant non-major bleeding in studies of anticoagulants in atrial fibrillation and venous thromboembolic disease in non-surgical patients: communication from the SSC of the ISTH. Journal of Thrombosis and Haemostasis. 2015 Nov;13(11):2119-26.

18. Wells G, Shea B, O'Connell D, Peterson J, Welch V, Losos M, et al. The Newcastle-Ottawa Scale (NOS) for assessing the quality of nonrandomised studies in meta-analyses [Internet]. [cited $2020 \mathrm{Apr} 19$ ]. Available from: http://www.ohri.ca/programs/clinical_epidemiology/oxford.asp

19. Tierney JF, Stewart LA, Ghersi D, Burdett S, Sydes MR. Practical methods for incorporating summary time-to-event data into meta-analysis. Trials. 2007 Jun 7;8:16.

20. Antoniou GA, Antoniou SA, Smith CT. A guide on meta-analysis of time-to-event outcomes using aggregate data in vascular and endovascular surgery. Journal of Vascular Surgery. 2020 Mar 1;71(3):1002-5.

21. Higgins JPT, Thompson SG, Deeks JJ, Altman DG. Measuring inconsistency in meta-analyses. BMJ. 2003 Sep 6;327(7414):557-60. 
22. Easterbrook PJ, Berlin JA, Gopalan R, Matthews DR. Publication bias in clinical research. Lancet. 1991 Apr 13;337(8746):867-72.

23. DerSimonian R, Laird N. Meta-analysis in clinical trials. Control Clin Trials. 1986 Sep;7(3):17788.

24. Riley RD, Higgins JPT, Deeks JJ. Interpretation of random effects meta-analyses. BMJ [Internet]. 2011 Feb 10 [cited 2020 Jul 26];342. Available from: https://www.bmj.com/content/342/bmj.d549

25. Donegan S, Williamson P, D'Alessandro U, Tudur Smith C. Assessing key assumptions of network meta-analysis: a review of methods: Assessing assumptions of network meta-analysis. Research Synthesis Methods. 2013 Dec;4(4):291-323.

26. Harrer M, Cuijpers P, Ebert D. Doing Meta-Analysis in R. 2019 Jan 28 [cited 2020 Apr 23]; Available from: https://bookdown.org/MathiasHarrer/Doing_Meta_Analysis_in_R/

27. Guyatt G, Oxman AD, Akl EA, Kunz R, Vist G, Brozek J, et al. GRADE guidelines: 1. IntroductionGRADE evidence profiles and summary of findings tables. J Clin Epidemiol. 2011 Apr;64(4):383-94.

28. Guyatt GH, Oxman AD, Vist GE, Kunz R, Falck-Ytter Y, Alonso-Coello P, et al. GRADE: an emerging consensus on rating quality of evidence and strength of recommendations. BMJ. $2008 \mathrm{Apr}$ 26;336(7650):924-6.

29. Schünemann H, Hill S, Guyatt G, Akl EA, Ahmed F. The GRADE approach and Bradford Hill's criteria for causation. J Epidemiol Community Health. 2011 May;65(5):392-5.

\section{Figures}




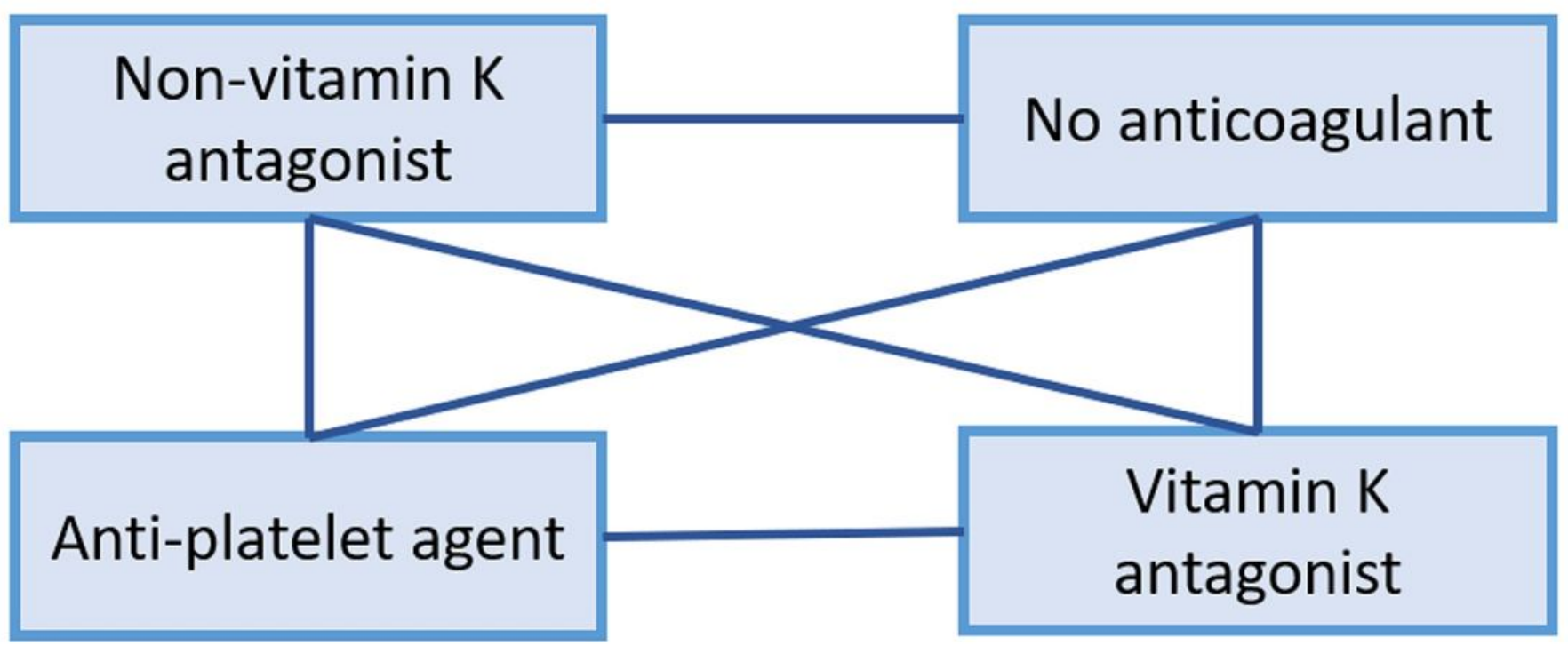

Figure 1

Potential comparisons between interventions within the network meta-analysis framework.

\section{Supplementary Files}

This is a list of supplementary files associated with this preprint. Click to download.

- Additionalfile1.docx

- Additionalfile2.docx 